Article

\title{
Hue-Angle Product for Low to Medium Spatial Resolution Optical Satellite Sensors
}

\author{
Hendrik Jan van der Woerd ${ }^{1, *(1)}$ and Marcel Robert Wernand ${ }^{2}$ (ID \\ 1 Institute for Environmental Studies (IVM), Water \& Climate Risk, VU University Amsterdam, \\ De Boelelaan 1087, Amsterdam 1081HV, The Netherlands \\ 2 Royal Netherlands Institute for Sea Research, Coastal Systems, Marine Optics \& Remote Sensing, \\ P.O. Box 59, Den Burg 1790AB, Texel, The Netherlands; marcel.wernand@nioz.nl \\ * Correspondence: h.j.vander.woerd@vu.nl; Tel.: +31-020-598-9555
}

Received: 15 November 2017; Accepted: 24 January 2018; Published: 26 January 2018

\begin{abstract}
In the European Citclops project, with a prime aim of developing new tools to involve citizens in the water quality monitoring of natural waters, colour was identified as a simple property that can be measured via a smartphone app and by dedicated low-cost instruments. In a recent paper, we demonstrated that colour, as expressed mainly by the hue angle $(\alpha)$, can also be derived accurately and consistently from the ocean colour satellite instruments that have observed the Earth since 1997. These instruments provide superior temporal coverage of natural waters, albeit at a reduced spatial resolution of $300 \mathrm{~m}$ at best. In this paper, the list of algorithms is extended to the very first ocean colour instrument, and the Moderate Resolution Imaging Spectroradiometer (MODIS) 500-m resolution product. In addition, we explore the potential of the hue angle derivation from multispectral imaging instruments with a higher spatial resolution but reduced spectral resolution: the European Space Agency (ESA) multispectral imager (MSI) on Sentinel-2 A,B, the Operational Land Imager (OLI) on the National Aeronautics and Space Administration (NASA) Landsat-8, and its precursor, the Enhanced Thematic Mapper Plus (ETM+) on Landsat-7. These medium-resolution imagers might play a role in an upscaling from point measurements to the typical 1-km pixel size from ocean colour instruments. As the parameter $\alpha$ (the colour hue angle) is fairly new to the community of water remote sensing scientists, we present examples of how colour can help in the image analysis in terms of water-quality products.
\end{abstract}

Keywords: hue; colour; CZCS; Landsat-7 and Landsat-8; MERIS; MODIS; MSI; OLCI; SeaWiFS

\section{Introduction}

Although ocean colour satellite sensors only measure the visual radiation in a limited number of narrow spectral bands, they are perfectly capable of measuring the colour of natural waters [1]. Colour is a sensation that originates in the human perception of radiation between the wavelengths of 380-720 nm. Since the beginning of the 20th century, the science of human colour perception has been well studied [2,3]. Since that time, colour has become more and more important in societal communication, as is evident from the development in colour printing, colour screens (television, computer monitors, tablets), digital cameras, and smartphones. Therefore, colour was chosen in the European Citclops (Citizens' observatories for coast and ocean optical monitoring) project [4] as a primary parameter to involve citizens in the monitoring of natural waters [5]. Involvement of the general public in the collection of observations of the environment has gained momentum under the term "citizen science". These observations might help scientists in their understanding of local processes, and create awareness and commitment in the environmental stewardship of the general public [6-8]. 
At the end of the 19th century, protocols were developed to provide consistent measures of "water colour" by using human perception to compare colours of natural waters to a predefined scale of colours [9]. One of these colour comparators is the Forel-Ule (FU) scale, a historical standard that has recently been recalibrated [10,11], and is still used to this day [12]. This colour-comparator scale was developed because of technological limitations that existed at the end of the 19th century. However, new initiatives in participatory science, such as those within the European Union (EU) Citclops project, indicate that the colour comparison methodology can be transferred to nowadays measuring techniques using smartphones [13], and made fully operational in the EyeOnWater colour application and supporting website (www.eyeonwater.org/color).

In Citclops, it was considered beneficial for multiple end-users to validate and compare the localised in-situ data collected with the Eye-On-Water App (EOW) with recent and historical maps of water composition and colour of a larger region, which are mainly based on satellite observations. A recent article by Busch et al. provided a demonstration of this integration for the Ebro Delta. [14]. Since no water colour product is offered by the standard processing from ESA and NASA, Van der Woerd and Wernand [1] published algorithms to derive the colour hue angle $(\alpha)$ and FU-scale number accurately and consistently from four spaceborne instruments: the Moderate Resolution Imaging Spectroradiometer (MODIS), the Sea-viewing Wide Field-of-view Sensor (SeaWiFS), Medium Resolution Imaging Spectrometer (MERIS), and the Ocean and Land Colour Instrument (OLCI) on the Sentinel-3 platform. The algorithms are now publicly available and implemented in the ESA Sentinel Application Platform (SNAP) open software [15].

Ocean colour measurements from space are well suited to assess dynamics in water composition over a broad range of spatial scales, from global ocean composites down to the basic pixel size, which is $300 \mathrm{~m}$ in the case of MERIS Full Resolution (FR) data. The colour of the open ocean varies mainly with the chlorophyll- $a$ concentration (CHL) in phytoplankton $[9,16]$. However, most EOW colour observations are collected close to the shore, where other components contribute to or dominate the colour of the water column, such as absorption by plant degradation products (Coloured Dissolved Organic Matter, or CDOM) and Suspended Particulate Matter concentration (SPM), which is an indicator of turbidity, coastal erosion, riverine flux, and wind or current generated resuspension [17].

Busch et al. [14] demonstrated that far from the shore in complex coastal waters (such as the Ebro Delta in northwest Spain), the derived colour of the MERIS FR (300 m) agreed well with the colour derived from hyperspectral data in optically deep waters. However, MERIS FR data were not of good quality closer to the shore, where the EoW App might provide the most abundant information. The degradation of satellite data near shore can be the result of influences by land reflection on atmospheric correction, sea-bottom reflection, and subscale variability in one pixel. Large gradients in water composition and a land-sea boundary in one pixel can contribute to the uncertainty in the retrieval process. Wang et al. [18] came up with another solution to the large pixel problem by using the 500-m resolution product from MODIS, which was initially developed for land applications. It uses the bands at $466 \mathrm{~nm}, 553 \mathrm{~nm}$, and $647 \mathrm{~nm}$ (No. 3, 4, and 1, respectively), instead of the MODIS bands $8-14$ that are measured at a smaller spatial resolution $(1 \mathrm{~km})$.

Maybe a different type of sensor can play a role here: the classical land-oriented imagers such as Landsat observe the Earth with a spatial resolution in the order of $30 \mathrm{~m}$. These instruments have less spectral resolution than the ocean colour instruments (see Figure 1); the number of bands in the visual domain is limited (3-5); and also, each band covers a wide range of wavelengths, much wider than the characteristic 10-nm Full Width at Half Maximum (FWHM) of instruments such as MERIS, MODIS, and OLCI. Nevertheless, these imagers have been used in many studies of inland and coastal waters, some with success [19-22].

The aim of this paper is to extent the hue angle $(\alpha)$ algorithms for 10 spaceborne instruments that monitor or have monitored the natural waters on Earth. In Van der Woerd and Wernand [1], it was already demonstrated that $(\alpha)$ can be derived consistently form SeaWiFS, MODIS, MERIS, and OLCI, facilitating the future consistent merging of these four sensors to a global monitoring of colour from 
1997 to the present (see also Van der Woerd et al. [23]). In this paper, we present results for two other ocean colour sensors: the MODIS 500-m product, and the observations by the Coastal Zone Color Scanner (CZCS), allowing the analysis of measurements over the years 1979-1986 [24]. In this paper, we also investigate the accuracy of the hue angle retrieval from two present medium-resolution imagers: the multispectral imager (MSI) on Sentinel-2 A,B [25], and the Operational Land Imager (OLI) on Landsat-8 [26]. In order to allow a coupling to historical data, the Landsat-8 precursors Landsat-7 ETM is also analysed. Finally, in order to demonstrate and discuss the merit of the true colour products from these 10 satellites, some general coupling of $(\alpha)$ to CHL and the absorption at $440 \mathrm{~nm}$ is presented.

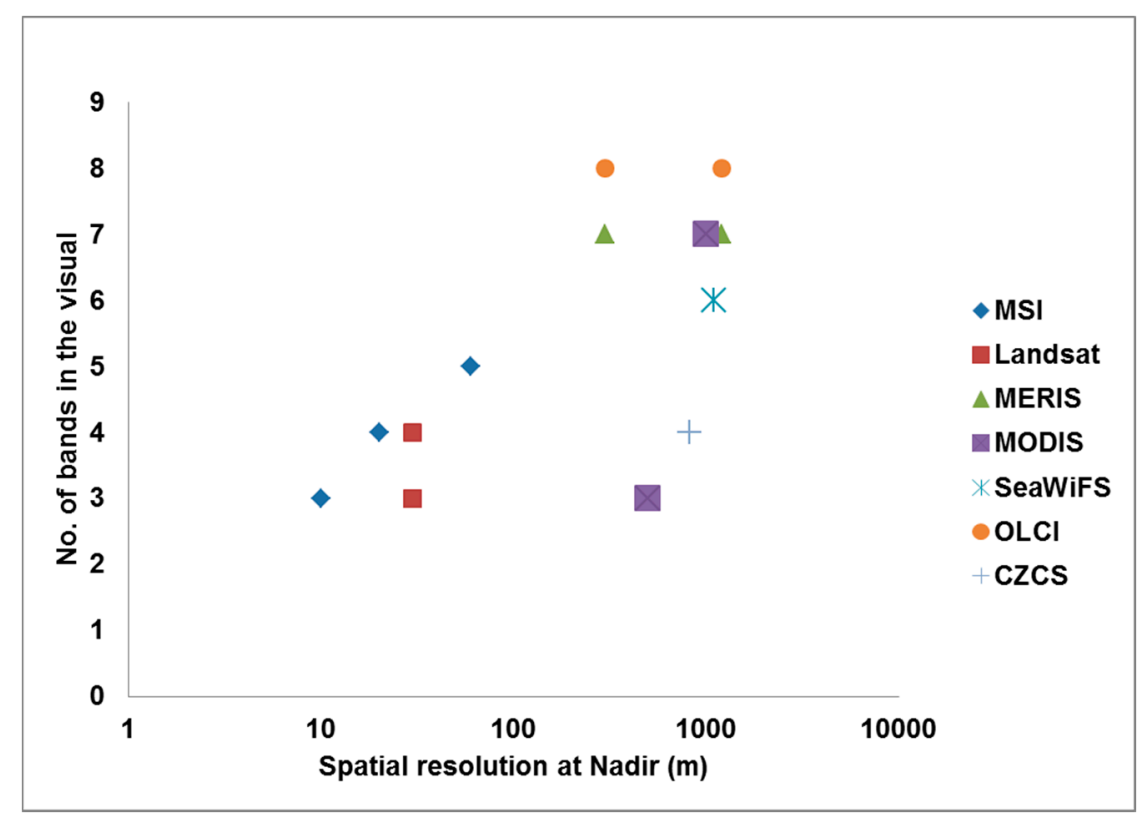

Figure 1. Plot of the available spectral bands in the visual range $(400-710 \mathrm{~nm})$ as function of spatial resolution for seven types of sensors. The multispectral imager (MSI) provides three different resolution modes, Landsat 8 has one band more than Landsat 7 at 30-m resolution. The Medium Resolution Imaging Spectrometer (MERIS), Moderate Resolution Imaging Spectroradiometer (MODIS) and Ocean and Land Colour Instrument (OLCI) provide products at two resolutions.

\section{Materials and Methods}

\subsection{Satellite Colourimetry}

The derivation of the colour of natural waters is based on the calculation of the so-called "Tristimulus" values of the three primaries $(X, Y, Z)$ that specify the colour stimulus of the human eye [4]. We start with the satellite remote sensing reflectance (Rrs), which is often derived from standard image processing. Rrs is a function of wavelength $(\lambda)$, and already corrected for atmospheric influences, the air-water interface, and illumination conditions; it is a quasi-inherent optical property [27]. The tristimulus values are defined by (example given for red):

$$
X=\int \operatorname{Rrs}(\lambda) \bar{x}(\lambda) d \lambda
$$

The standard colourimetry two degree Colour Matching Functions (CMFs) are represented by $\bar{x}$ (red), $y$ (green), and $\bar{z}$ (blue) (see Figure 2). The CMFs serve as weighting functions for the determination of the tristimulus values [3]. As ocean colour satellites do not provide hyperspectral coverage, the full spectrum should be first reconstructed, for example by linear interpolation, based on the Rrs measured 
at the spectral bands available. It was shown in Van der Woerd and Wernand [1] that for ocean-colour instruments, for example MERIS with nine bands, Equation (1) can be approximated by Equation (2).

$$
X=\sum_{i=1}^{i=9} M(i) \operatorname{Rrs}(i)
$$

This implies that each stimulus value can be calculated as a linear weighted sum of the band information. $M(i)$ are the linear coefficients that are precalculated for each of the three stimulus values and each instrument. Basically, $M(i)$ is the integral between band $(i-1)$ and $(i+1)$ of the tristimulus values times the weight $(w)$, which we assume is a linear function of the wavelength between the band considered $(w=1)$ and the previous or next band $(w=0)$. Suppose we want to calculate the integral of Equation (1) between band 1 at $\lambda_{1}$ and band 2 at $\lambda_{2}$, we can approach the integral by the summation

$$
X=\sum_{\lambda 1}^{\lambda 2} \operatorname{Rrs}(\lambda) \bar{x}(\lambda) d \lambda
$$

The next step is that we approach the $\operatorname{Rrs}(\lambda)$ with $\left(\lambda_{1} \leq \lambda \leq \lambda_{2}\right)$ by linear interpolation, such that

$$
\operatorname{Rrs}(\lambda)=\operatorname{Rrs}\left(\lambda_{1}\right) \times\left(\lambda-\lambda_{2}\right) /\left(\lambda_{1}-\lambda_{2}\right)-\operatorname{Rrs}\left(\lambda_{2}\right) \times\left(\lambda-\lambda_{1}\right) /\left(\lambda_{1}-\lambda_{2}\right)
$$

Now, if we combine Equations (3) and (4), we see that the summation is split in two components:

$$
X=\operatorname{Rrs}(\lambda 1) \sum_{\lambda 1}^{\lambda 2} \bar{x}(\lambda) \frac{(\lambda-\lambda 2)}{(\lambda 1-\lambda 2)} d \lambda-\operatorname{Rrs}(\lambda 2) \sum_{\lambda 1}^{\lambda 2} \bar{x}(\lambda) \frac{(\lambda-\lambda 1)}{(\lambda 1-\lambda 2)} d \lambda
$$

Since we know the CMF, the summation can be made once we know which two bands to choose. In this example, the total weight at band $2(M(2))$ consists of the summation in Equation (5) with the previous band at a shorter wavelength $\left(\lambda_{1}\right)$, and the next band at a larger wavelength $\left(\lambda_{3}\right)$.

Once the tristimulus values $(X, Y, Z)$ have been calculated, the three values are normalised, and the colour is expressed in the chromaticity coordinates $(x, y)$ :

$$
x=\frac{X}{X+Y+Z} y=\frac{Y}{X+Y+Z}
$$

The white point has the coordinates $x_{w}=y_{w}=1 / 3$. In the $(x, y)$ chromaticity plane, the coordinates are transformed to polar coordinates with respect to the white point, and the hue angle is derived. The hue angle $(\alpha)$ lies between the vector to a point with coordinates $\left(x-x_{w}, y-y_{w}\right)$ and the positive $\mathrm{x}$-axis (at $y-y_{w}=0$ ), giving higher angles in an anti-clockwise direction (see Figure 2 in Van der Woerd and Wernand [1]). Note that the definition of $(\alpha)$ is different in Wang et al. [18].

$$
\alpha=\arctan \left(y-y_{W}, x-x_{W}\right) \text { modulus } 2 \pi
$$

Finally, each $(\alpha)$ is corrected by a small number $(\Delta)$ to get the best linear relation between the calculated hyperspectral hue angle and the ocean colour instrument hue angle. This correction $(\Delta)$ compensates for the spectra of natural waters deviating from the linear interpolation between detector bands. This is illustrated in Figure 2, which compares the hyperspectral Rrs spectrum and reconstructed spectrum for open ocean water $\left(\mathrm{CHL}=0.015 \mathrm{mgm}^{-3}\right)$, based on the MERIS information in nine spectral bands. 


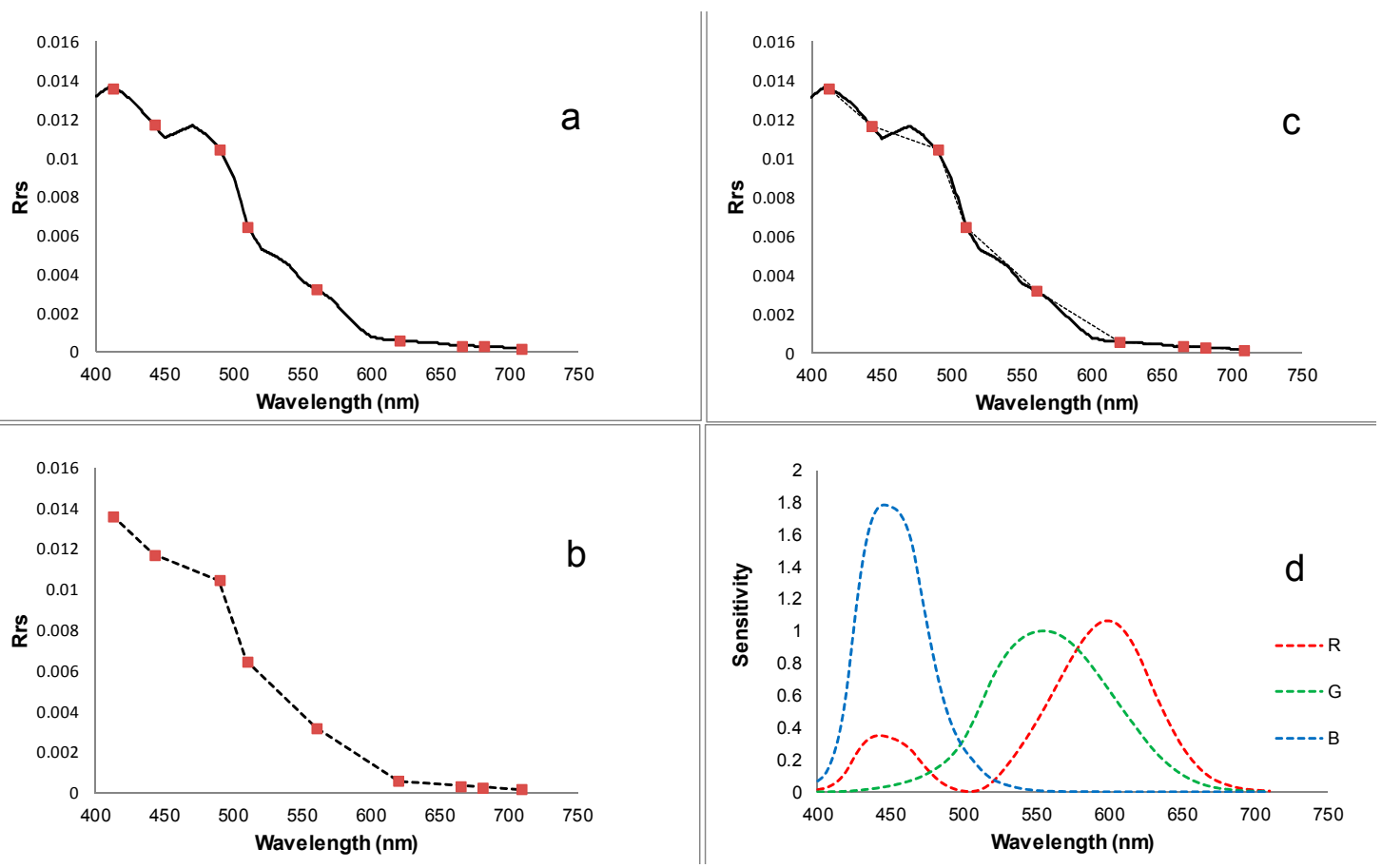

Figure 2. Original hyperspectral data plus red squares at the position of MERIS bands (a); the reconstructed spectrum from Rrs at nine MERIS spectral bands (b); a comparison of the two (c); and Colour Matching Function (CMF) curves for red, green, and blue that weight the spectral information to derive the hue angle $(\mathbf{d})$.

\subsection{Synthetic and Field Spectra}

As a benchmark for algorithm development, we use the synthetic remote sensing reflectance data from the International Ocean Colour Coordinating Group (IOCCG) in this study [17]. The above water Rrs is simulated using Hydrolight [28] and a set of inherent optical properties derived from field measurements, representing a wide range of compositions that occur in natural waters. Details on this data set can be found in Lee et al. [17] and the IOCCG website [29]. The 500 synthetic Rrs spectra are provided every $10 \mathrm{~nm}$ between $400-800 \mathrm{~nm}$. In this paper, we refer to the "true" or "hyperspectral" colour when these spectra are interpolated on a 1-nm grid and the hue angle $(\alpha)$ is calculated based on Equations (1) and (3).

Validation of the algorithms is carried out by analysis of a new hyperspectral Rrs in-situ data set, based on field measurements collected during the EU Citclops project [4]. Validation is carried out by comparing the hyperspectral hue angle to the sensor hue angle. The sensor hue angle is calculated from the extraction of Rrs at the spectral band positions of each sensor. A total of 603 spectra were accumulated in 2013 at 43 sampling stations at the North Sea and Dutch coastal and inland water bodies. The concentrations of the water quality indicators varied considerably among the 43 stations, reflecting the widely different water body types that were sampled: clear North Sea $\left(\mathrm{CHL}<0.5 \mathrm{mgm}^{-3}\right)$, turbid coastal North Sea (SPM up to $24 \mathrm{gm}^{-3}$ ), rivers, eutrophic lakes (CHL up to $55 \mathrm{mgm}^{-3}$ ), and even peat lakes with extreme CDOM values ( $\mathrm{g} 440$ up to $3.1 \mathrm{~m}^{-1}$ ). More information can be found in Novoa et al. [13]. At each station, hyperspectral measurements were carried out using TriOS-RAMSES hyperspectral radiometers following the NASA protocols [30]. The measurements included sky radiance $\left(\mathrm{L}_{\mathrm{sky}}\right)$, upwelling radiance $\left(\mathrm{L}_{\mathrm{sfc}}\right)$, and incident spectral irradiance $\left(\mathrm{E}_{\mathrm{s}}\right)$. The radiometers cover the spectral range 320-950 nm, with a spectral resolution of $3.3 \mathrm{~nm}$ (Full Width at Half Maximum, 
or FWHM) and an accuracy of $0.3 \mathrm{~nm}$. The water-leaving radiance $\mathrm{L}_{\mathrm{w}}(\lambda, 0+)$ at wavelength $(\lambda)$ just above the surface $(0+)$ is derived from Equation (8):

$$
\mathrm{L}_{\mathrm{W}}(\lambda, 0+)=\mathrm{L}_{\text {sfc }}(\lambda, 0+)-\rho \times \mathrm{L}_{\text {sky }}(\lambda)
$$

The reflectance factor $\rho$ is a correction factor to compensate for Fresnel reflectance at the air/water boundary [30], which is defined as the fraction of skylight actually reflected from the wave roughened (water) surface. In this study, we simply calculated $\rho$ by demanding that the water-leaving radiance at $360 \mathrm{~nm}$ equals zero. The intrinsic colour of the water is given by the spectral distribution of the remote-sensing reflectance $\operatorname{Rrs}(\lambda, 0+)$ that is calculated as the ratio of water-leaving radiance $\mathrm{L}_{W}(\lambda, 0+)$ over downwelling irradiance $\mathrm{E}_{\mathrm{S}}(\lambda, 0+)$ :

$$
\operatorname{Rrs}=\mathrm{L}_{\mathrm{W}}(\lambda, 0+) / \mathrm{E}_{\mathrm{S}}(\lambda, 0+)
$$

Again, the intrinsic or true colour was calculated by conversion of the TriOS data to a 1-nm grid, and calculation of the hyperspectral hue angle by Equations (1), (6), and (7).

\subsection{Satellite Simulated Spectra}

In Van der Woerd and Wernand [1], the hue-angle algorithm was based on the 500 simulated Rrs spectra for SeaWiFS, MODIS, MERIS, and OLCI. First, the weights $M(i)$ of Equation (2) were precalculated based on CMFs and the central wavelengths of the bands of each sensor. We refer to Van der Woerd and Wernand [1] for details. It was essential that these sensors have 10-nm narrow bands, making sure that each band reliably measures the actual Rrs at that wavelength, since the variations of Rrs within the band are small. Subsequently, the offset $(\Delta)$ for all of the hue angles in the interval 30-230 degrees is determined by comparison of the reconstructed sensor spectrum (Equation (2)) to the original hyperspectral spectrum (Equation (1)).

In this paper, we extend the list of imaging sensors with instruments that all have broad spectral bands with a radiation detection efficiency that is not uniform within each band. This detection efficiency is described by the Spectral Response Function (SRF). For example, Figure 3 shows the SRF for the five bands from the MSI sensor on Sentinel-2 [31]. Similar functions were used for Landsat-8 [32] and Landsat-7 ETM+ [32]. The MODIS 500-m product uses only three wide spectral bands [33], which are different from the standard MODIS band setting that is used for ocean-colour imaging, but prove to be useful for analysing smaller waters (Wang et al., 2015). The CZCS is the predecessor of the present ocean-colour instruments, and has a limited set of four narrow spectral bands, plus a broad band in the Near-Infrared (NIR) [34]. 


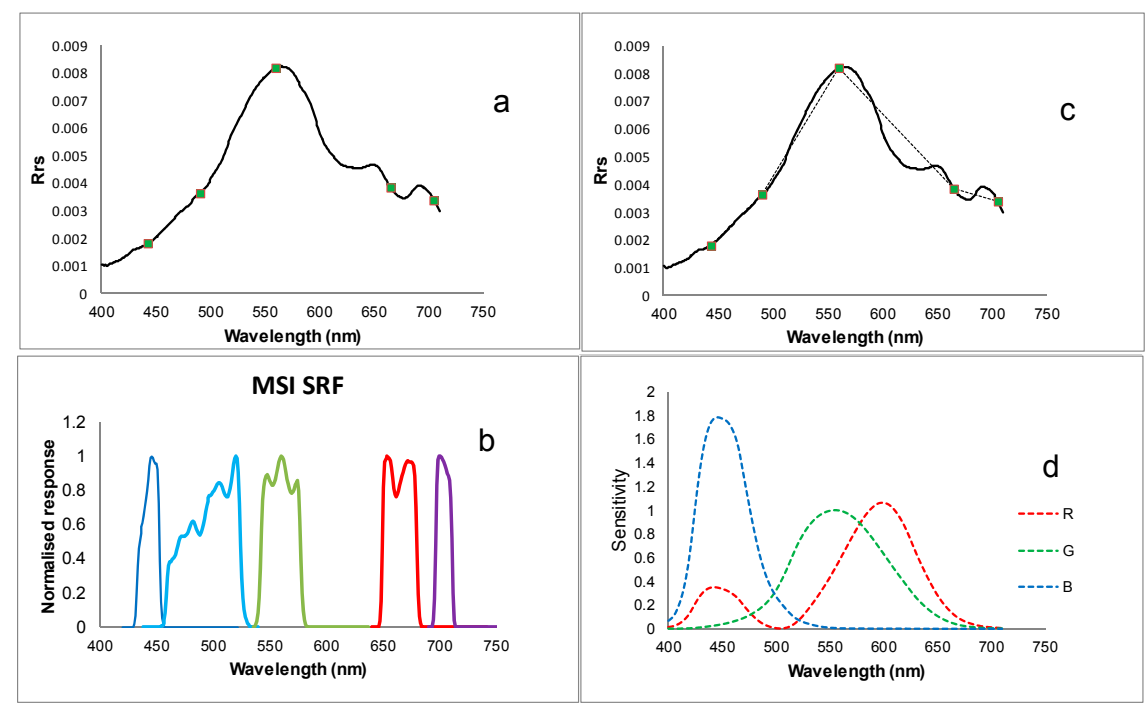

Figure 3. Original hyperspectral data plus green squares at the central positions of MSI bands (a); MSI measures the visual spectrum in only five wide bands (b); and the reconstructed spectrum will start to deviate from the original spectrum, especially at green and red wavelengths (c,d).

In case of the ocean-colour instruments, such as MERIS, the number of bands, the bandwidth, and band positions are such that the full spectrum is reasonably well reconstructed. Therefore, the weighting with the CMF curves is accurate enough to derive the hue angle $(\alpha)$ over the entire range of natural water colours (see Figure 4). However, in the case of a limited set of broad bands (Figure 3), it is not possible to reconstruct the underlying Rrs spectrum with high accuracy. In other words, the band averaging of radiation introduces a larger uncertainty in the folding with the CMF curves. We have approached this problem as follows: the weights $M$ (see Equation (2)) were precalculated based on the mean wavelength of each band, i.e., a linear interpolation between the centres of the broad bands. All of the 500 IOCCG spectra and fields spectra were folded with the SRF of the sensor bands to derive the Rrs that is measured at each band, and the hue angle is derived from Equations (2) and (3). The results are compared with the hue angle of the original hyperspectral data.

\section{Results}

\subsection{Sensor Algorithms}

The coefficients $M(i)$ of eight sensor configurations are given in Table 1. The entries for MERIS, MODIS, SeaWiFS, and OLCI on Sentinel-3 have already been published in Van der Woerd and Wernand [1], and the MERIS result serves here as a benchmark for the new calculations. Each entry is composed as follows: the columns are identified by the central wavelength of each band in $\mathrm{nm}(\mathrm{R} 400, \mathrm{R} 412, \ldots)$ ). In the second row, the band number is provided, as given in the product documentation of each instrument. The next three rows contain the coefficients for red $(X)$, green $(Y)$, and blue (Z). Note that all of the instruments start with R400 and end with R710, because these are the end points of the spectrum reconstruction. Outside this visual interval, the CMF values are near to zero. The entries at R400 and R710 do give relevant information, because they complete the picture of how well the sensor bands are able to measure the three primary colours. For example, MERIS has bands at $413 \mathrm{~nm}$ and $708 \mathrm{~nm}$, and therefore covers all of the colours very well. The R400 and R710 values are less than $1 \%$ of the total information in $X, Y$, and $Z$. However, if we consider the blue $(Z)$ R400 value of the CZCS, it becomes clear that a large amount (10.745) is not detected by the CZCS, which has a first blue band at $443 \mathrm{~nm}$. The importance of this number depends on the underlying spectrum, for if its radiation at $400 \mathrm{~nm}$ is zero, there is no effect. However, if the radiation at $400 \mathrm{~nm}$ is relatively high, we can expect that the CZCS has problems in retrieving the proper hue angle. For all 
of the instruments, the weights are such that in case the Rrs is constant, (wavelength-independent or white) the sum of all of the weights is $(106.665 ; 106.824,106.335)$, and the $(x, y)$ values are $(0.3335$; $0.3340)$ close to the theoretical white point of $(1 / 3,1 / 3)$.

Figure 4 shows the results of the hue angle calculations for six sensors. At the horizontal axis, this sensor hue angle is plotted, as derived from Equations (2), (6), and (7)). At the vertical axis, the deviation from the hyperspectral hue angle (Equations (1), (6), and (7)) is provided. This $\Delta$ is defined as the hyperspectral $(\alpha)$-sensor $(\alpha)$, and is expressed in degrees $\left(^{\circ}\right)$. The shape of the offset $(\Delta)$ is more or less similar in for all of the sensors: a bell-shaped curve that has the largest offset in the green part of the spectra around $(\alpha) 125$ degrees. On the one hand, it is expected that the sensors with a limited set of bands show larger offsets than an instruments such as MERIS, with nine bands situated at well-chosen wavelengths to cover the dynamics in water-leaving spectra. On the other hand, the offset is not completely random, and as a first approximation, the correction $(\Delta)$ can be described by a fifth order polynomial, which is indicated by the drawn blue line. If (a) is the calculated hue angle divided by 100 , $\Delta$ can be approximated (for MERIS) by:

$$
\Delta=-12.05 a^{5}+88.93 a^{4}-244.70 a^{3}+305.241 a^{2}-164.70 a+28.53
$$

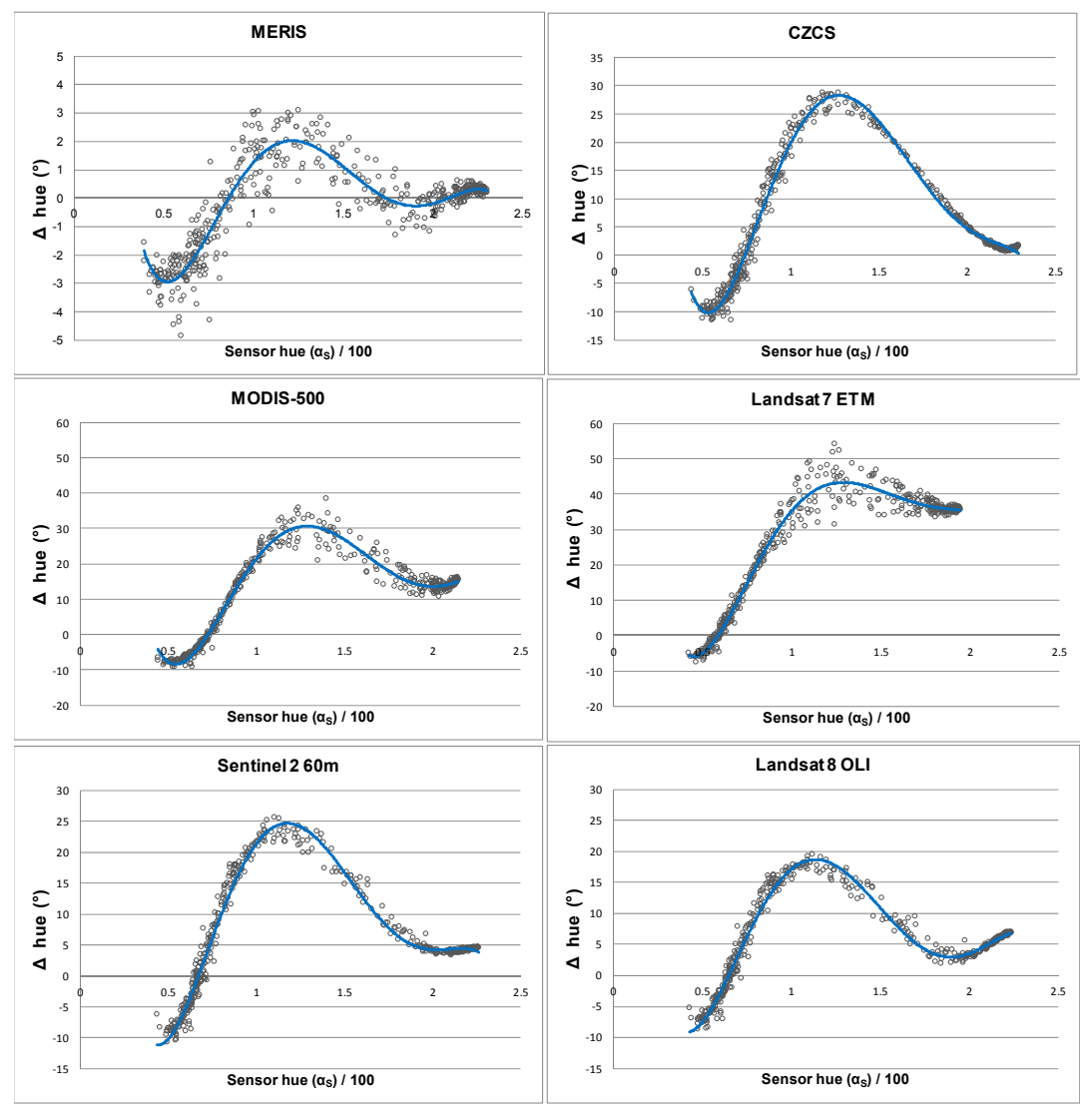

Figure 4. Deviation in degrees from the hyperspectral hue angle for six instruments. The horizontal axis gives the hue angle based on the reconstructed spectra (scaled by 100 for practical reasons). Note that, although the shape follows the same pattern for all of the instruments, the vertical scale is very different. The drawn line is the fifth order polynomial fit to the data.

The best approximation of the true colour of water is reached by adding this $\Delta$ correction to the results of Equations (2) and (3). In Table 2, the polynomial coefficients are given for all of the instrument configurations. 
Table 1. Linear coefficients to calculate the chromaticity values $(X, Y, Z)$ for eight sensor configurations.

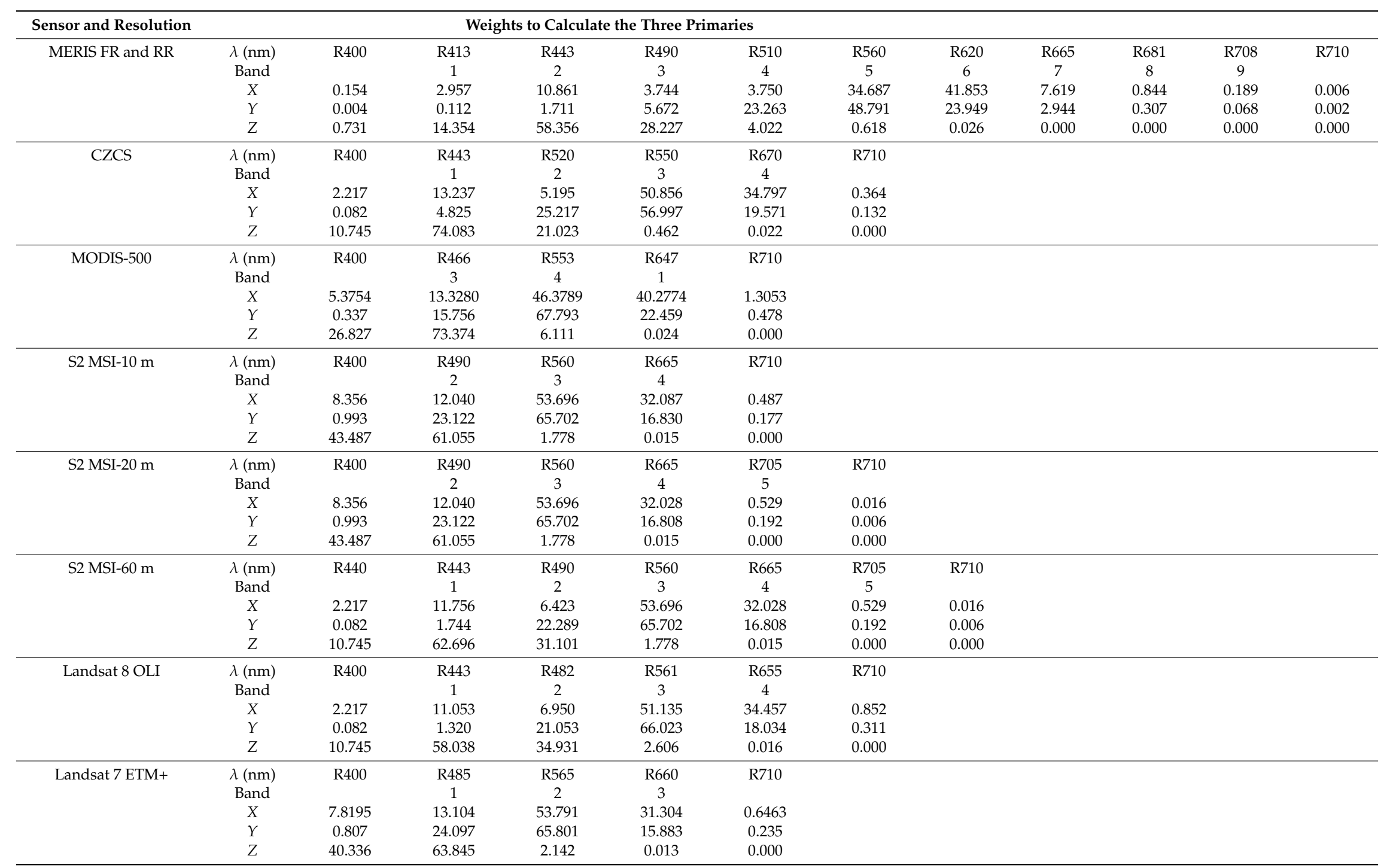


Table 2. Polynomial coefficients to calculate the hue-angle offset $(\Delta)$ for each instrument.

\begin{tabular}{|c|c|c|c|c|c|c|c|}
\hline \multirow{2}{*}{ Sensor } & \multirow{2}{*}{ Resolution (m) } & \multicolumn{3}{|c|}{ Polynomial Coefficients } & \multirow[b]{2}{*}{$a^{2}$} & \multirow[b]{2}{*}{$\mathbf{a}$} & \multirow{2}{*}{ Constant } \\
\hline & & $a^{5}$ & $a^{4}$ & $a^{3}$ & & & \\
\hline MERIS FR and RR & 300 and 1200 & -12.05 & 88.93 & -244.70 & 305.24 & -164.70 & 28.53 \\
\hline CZCS & 1100 & -65.95 & 510.37 & -1475.80 & 1927.61 & -1078.62 & 202.25 \\
\hline MODIS-500 & 500 & -68.36 & 534.04 & -1552.76 & 2042.42 & -1157.00 & 223.04 \\
\hline S2 MSI-10 m & 10 & -164.83 & 1139.90 & -3006.04 & 3677.75 & -1979.71 & 371.38 \\
\hline S2 MSI-20 m & 20 & -161.23 & 1117.08 & -2950.14 & 3612.17 & -1943.57 & 364.28 \\
\hline S2 MSI-60 m & 60 & -65.74 & 477.16 & -1279.99 & 1524.96 & -751.59 & 116.56 \\
\hline Landsat 8 OLI & 30 & -52.16 & 373.81 & -981.83 & 1134.19 & -533.61 & 76.72 \\
\hline Landsat 7 ETM+ & 30 & -84.94 & 594.17 & -1559.86 & 1852.50 & -918.11 & 151.49 \\
\hline
\end{tabular}




\subsection{Algorithm Validation}

The set of Rrs spectra from the central North Sea and Dutch coastal and inland waters were used to validate the hue-angle algorithms. As these hyperspectral data are real measurements, it is expected that measurement errors and variation in water types within the sample will produce larger errors as compared to the modelled IOCCG dataset. Again, the hyperspectral hue angle was calculated and compared with the calculated sensor hue angle. Note that also for these spectra, the SRF of all of the bands was first folded with the spectrum before Equations (2) and (3) were applied, and corrected with the sensor-specific $\Delta$-polynomial. Figure 5 shows that this algorithm is able to derive the true colour in a consistent manner over the full range of potential angles. The slope is close to 1.00, and the offset remains small. However, what is different is that the scatter around the 1:1 line increases significantly for sensors such as MODIS-500 and the land imagers Landsat 7 and 8, notably in the green region $\left(70^{\circ}-130^{\circ}\right)$. This demonstrates that variations in the underlying hyperspectral distribution have a much larger effect on the hue angle retrieval for sensors with limited and wide bands.

\subsection{Accuracy}

In this study, we have two datasets available that can be used to characterise the accuracy of $(\alpha)$ retrieval; the 500 IOCCG spectra, and the 603 field spectra. Figures 4 and 5 show that the accuracy is mainly a function of $(\alpha)$ itself. Each sensor has intervals in $(\alpha)$ that can be covered perfectly with that particular band setting (such as blue waters in Sentinel 2 MSI), or can only be poorly covered (such as green waters with Landsat-7 ETM). Therefore, we present in Figure 6 the standard deviation in both datasets in intervals of 30 degrees. The standard deviation is defined for the parameter (sensor- $\alpha$ minus hyperspectral- $\alpha$ ). Note that the results are provided for the interval $37^{\circ}-230^{\circ}$ degrees where the fifth order polynomial was fitted.

From Figure 6, it can be concluded that MERIS and OLCI perform well over the entire range, with an average standard deviation for the IOCCG data of around one degree. The other classic ocean colour sensors-CZCS, SeaWiFS, and MODIS-perform almost as well in the extreme blue waters $\left(\alpha>170^{\circ}\right)$, but have a standard deviation around two degrees for the green-brown colours $\left(\alpha<140^{\circ}\right)$. The MODIS-500 and Landsat ETM sensors, both three-band instruments, have problems in covering the whole colour scale. Already, when $\left(\alpha>110^{\circ}\right)$, the band information plus the large polynomial correction (in the order of 40 degrees) introduce large errors in the hue-angle calculation. As stated above, field data contain multiple error sources (clouds, sky correction, waves), and indeed the lower panel in Figure 6 shows larger standard deviations than the IOCCG data. First of all, we must emphasise that the two intervals $\left(170^{\circ}-200^{\circ}\right.$ and $\left.200^{\circ}-230^{\circ}\right)$ are not representative because of the limited number of spectra. This number $(\mathrm{N}=)$ is given below the interval. However, the field data contain a wealth of information at all other hue-angle intervals. The hue-angle dependent standard deviation of the instruments MERIS, OLCI, MODIS, and SeaWiFS follows similar patterns as for the modelled data, but scaled up by a factor of two. Also, the CZCS, OLI, and MSI do very well, and have an average standard deviation of $4^{\circ}$ to $5^{\circ}$. The three-band instruments again show larger errors with increasing hue angles, and seem to be quite inaccurate for all ocean-type waters. However, more and better data of these blue waters should be used to test the performance of these two sensors in that range. 


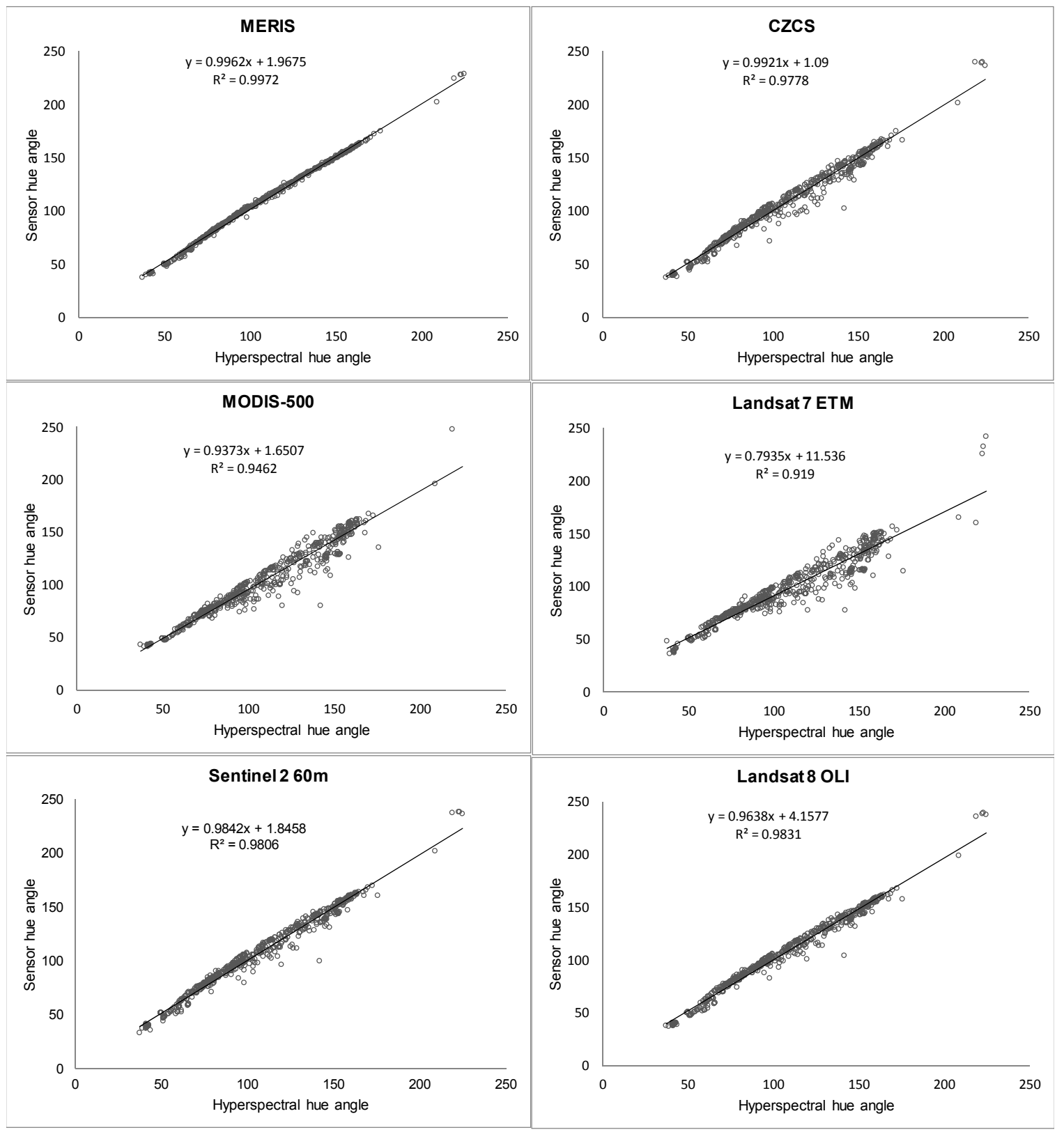

Figure 5. Validation of the hue angle reconstruction for six instruments. 

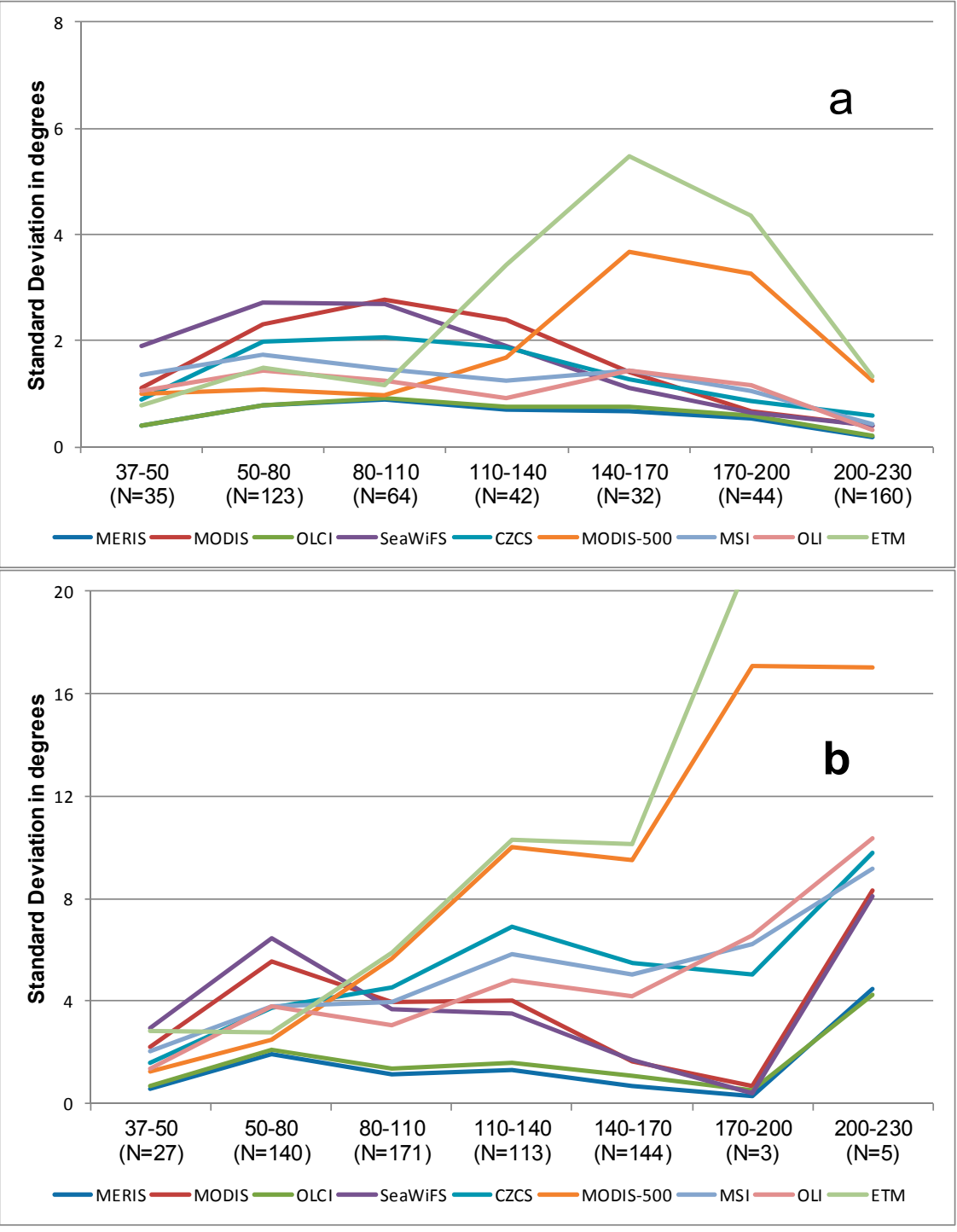

Figure 6. Plot of the standard deviation in the parameter ( $\alpha$-sensor minus $\alpha$-hyperspectral) for nine sensors. On the horizontal axis, the hyperspectral hue interval is given in degrees, together with the number of spectra $(\mathrm{N})$ that were used to calculate the standard deviation. The upper panel (a) is based on the International Ocean Colour Coordinating Group (IOCCG) data, and the lower panel (b) is based on the field data.

\subsection{The Use of Hue}

After the retrieval potential of the hue angle by medium-resolution imaging instruments is presented and characterised, it is important to introduce the value of this parameter, and how it can be used in the study of biology and ecology of oceans, lakes, and estuaries. First of all, it is a parameter that can be monitored over time to describe daily to seasonal changes and trends [9]. In Wernand et al. [9], it was already demonstrated that for open ocean waters (FU number 1 to 9), the colour is related to the $\mathrm{CHL}$ concentration. In the open ocean, $\mathrm{CDOM}$ co-varies with $\mathrm{CHL}$, and therefore, the $\mathrm{CHL}-(\alpha)$ relation holds [16]. In Figure 7, the relation between CHL and $(\alpha)$ is presented for the full range of angles, based on the simulations as described by the IOOCG [17]. Most prominent in the colouration of natural waters is the combined CHL and CDOM absorption of blue light, plus disturbance by absorption and scattering of SPM. The data in Figure 7 have been split into eight classes of CDOM concentration. This helps in the interpretation of the CHL- $(\alpha)$ relationship for natural waters. 
CDOM consists of very stable molecules that only degrade slowly in the natural environment [35]. This makes it a rather well kept quantity that varies little in oceans, seas, and estuaries. For example, the North Sea CDOM varies between 0.2 and $0.5 \mathrm{~m}^{-1}$ at $440 \mathrm{~nm}$, and we expect that the CHL- $(\alpha)$ relation for that continental shelf sea follows the orange dots in Figure 7 . The scatter in this relation is due to CDOM variations in the interval, plus SPM variations. The second major indicator of ecosystem health is the indication of light attenuation in the water. Figure 8 gives a quick impression of the relation between total absorption at $440 \mathrm{~nm}$ and $(\alpha)$. Although the vertical scale is logarithmic and the scatter is large, the underlying pattern in clearly visible. In order to get a much more detailed derivation of light attenuation, the underlying absorption and scattering properties must first be derived from the spectrum [36].

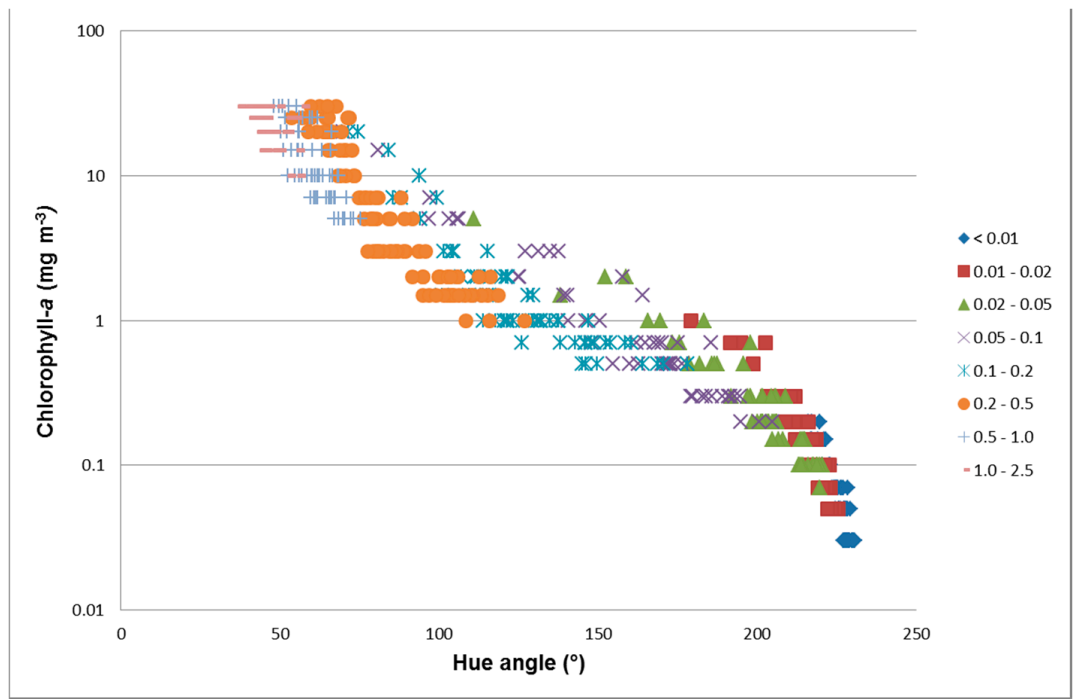

Figure 7. Relation between the hue angle and chlorophyll- $a$ concentration (CHL) for eight classes of Coloured Dissolved Organic Matter (CDOM) concentration. The first four classes $(0.001<\mathrm{g} 440<0.1)$ can be found in the open ocean, while the next two $(0.1<\mathrm{g} 440<0.5)$ represent regional seas and coastal waters. The very CDOM-rich waters $(0.5<\mathrm{g} 440<2.5)$ can be found in rivers and lakes.

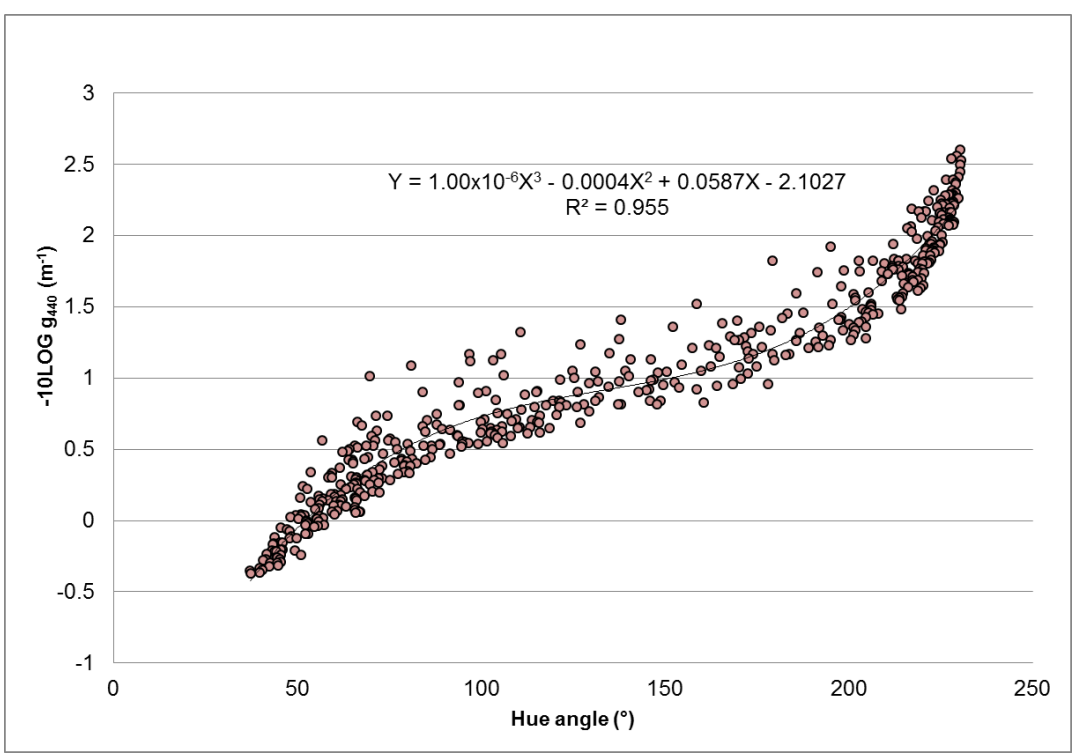

Figure 8. Relation between the hue angle and the absorption (a) of radiation at $440 \mathrm{~nm}$. 


\section{Discussion}

The information provided in Figures 4-6 provide a first impression of the potential for hue-angle retrieval from a range of optical satellite sensors. Although long underestimated, the CZCS sensor proved to be a worthy predecessor of the present ocean colour instruments. Although it was limited to only four narrow bands in the visual, and a wide band in the NIR, the ability to retrieve the hue angle was surprisingly good for most angles. In a sense, the CZCS is very similar in its optical band setting to SeaWiFS, but has limited information in the NIR, a region so important for atmospheric correction. Therefore, the CZCS has had limited success above turbid coastal and inland waters, but is important for the history of colour [9] and trend detection [24]. The error analysis presented in Figure 6 shows that the ESA (MERIS and OLCI) and NASA (SeaWiFS and MODIS) remain different in their accuracy to retrieve the hue for all of the waters with a hue of less than 150 degrees, corresponding with $\mathrm{FU}=7$ [10].

Wang et al. [18] made the innovative move to use the 500-m resolution product from MODIS (MOD09 and MYD09 formats) that is derived from three broad bands in the visual (No. 3, 4, and 1). Many arguments and calculations presented in Wang et al. [18] are very similar to the algorithms presented here. There are some differences that should be pointed out: first of all, the hue angle in this paper referenced the $y=1 / 3$ line in the $x y$-chromaticity diagram, while Wang et al. (2015) take the $x=1 / 3$ axis as zero $\left(\alpha=0^{\circ}\right)$. Second, they refer to the dominant wavelength (see their Figure 4 or any reference book). Third, do they analyse spectra with a dominant wavelength in the interval 555-575 $\mathrm{nm}$, which is equivalent to our definition of $\left(45^{\circ}<\alpha<75^{\circ}\right)$. Fourth, they use a linear combination of bands to calculate the hue angle, plus a small correction that is very similar in magnitude to our $\Delta$-correction (See Figure 4). We therefore seem to confirm their results, but also found that for waters with $\left(\alpha>110^{\circ}\right)$, the limitations of the band setting will introduce large errors; see Figures 4-6.

The Landsat 7 ETM+ sensor is designed to optimise the observation of land use and land cover, although recent studies [37] reveal that it also performs well in the monitoring of permanent and temporal variation in the surface of water bodies. In this paper, we have shown that its capacity to observe the composition in the water is clearly worse than all of the other sensors. Some reconstruction of $(\alpha)$ is possible, but the errors are large (Figures 4-6). This instrument does have a good track record on the observation of SPM [38], but this based on the absolute Rrs value of the green band around $565 \mathrm{~nm}$, not the radiation distribution. Van Hellemont and Ruddick [21] showed that OLI on Landsat 8 is even better suited to observe the SPM patterns in the coastal zone. In this paper, we have also shown that it performs well in $\alpha$-reconstruction. Both Landsat-7 and Landsat- 8 miss the ocean colour band setting to retrieve separately the concentration of CDOM, CHL, and SPM in the water at 30-m resolution. However, the hue angle opens the opportunity to study the patterns and evolution of the hue angle. A combination with the in-situ measurements of these constituents might provide a first rough estimate of CHL patterns, as was shown in Figure 7.

In a way, the MSI on Sentinel-2 A,B is rather similar to OLI in its ability to retrieve the hue angle. Figures 4-6 show a remarkably good linear behaviour and low error budget for most natural waters. What is interesting is that three types of band settings are available that come at different spatial resolutions. In this paper, we have only presented the five-band results that formally come at a resolution of $60 \mathrm{~m}$. However, some basic results are given in Table 2 for if a different band setting is tested at higher spatial resolution. In the operational ESA software SNAP, it is possible to interpolate the observation at a resolution higher than $60 \mathrm{~m}$. The algorithm presented here is implemented in the latest version of the SNAP (V6.0) [15].

Now that we have linked the remote sensing reflectance to colour by means of the hue angle, we can ask ourselves: "what is the potential of this parameter?" The hue angle is an addition to the large suite of parameters that is presently retrieved from the reflection spectra of natural waters. Figures 7 and 8 show that, if we have some extra information on the CDOM concentrations of the water under study, some basic relations between hue and CHL can be used for converting optical imaging spectrometer data to images of the CHL distribution of lakes and seas. Especially, 
the medium-resolution images such as MSI on Sentinel-2 could in principle use this hue angle to derive CHL products. However, maybe the value of hue is more related to the quality control of the processing, the atmospheric correction in particular. Van der Woerd et al. [23] showed that errors in the atmospheric correction of a MERIS scene of the North Sea were easily detected in the hue map. This information can help to detect errors and identify the impact on the other satellite products, such as the algal-1 and algal-2 products in MERIS. We are looking forward to a hue-angle analysis of the images collected by all of the sensors that have been presented in this paper. Overall, when we think of climate change, and its possible influence on the colour of natural waters, we can now connect the present with the past through the calculation of the hue angle and FU number from the youngest to the oldest satellite colour sensor, and even back to the early 1900s.

\section{Conclusions}

The derivation of the hue angle of natural waters from satellite data is based on the assumption that the spectral shape of the remote sensing reflectance falls in a group of curves that is described by the set of 500 IOCCG synthetic spectra. Validation by a set of over 600 spectra from North Sea and Dutch inland waters confirmed this point, and the hue angles could be well reconstructed from a limited set of narrow spectral bands, mimicking the band setting of MERIS, MODIS, SeaWiFS, and OLCI. In this paper, we have extended the analysis to instruments that have fewer and wider spectral bands in the visual domain (400-710 nm). Again, we see that the hue colour can be derived, but not all of the colours can be derived with the same accuracy. This is mainly due to the band setting, and not so much the width of the bands. In simulations that involved only the mean wavelength of each band (not including the detailed SRF), more or less similar results were obtained (results not shown). This study started with the requirements that were defined by citizen science for natural waters. The freely available EyeOnWater Colour app provides point measurements of the colour that could very well be integrated with the high-temporal ocean-colour sensor data. Indeed, Busch et al. [14] made a test for the Ebro Delta, and they showed that such an integration is possible. However, in that same article, it also became clear that there is still a (spatial) gap to fill between the position of most app users (more than $90 \%$ of the received data are situated within $1 \mathrm{~km}$ from the shoreline), and the first valid pixel from the shoreline from these ocean-colour sensors. This paper shows that there is great potential to fill this gap with the new medium-resolution sensors Sentinel-2 MSI and Landsat 8 OLI. Of course, this study is based on model simulations and ground-truth spectra. The next step is the testing of the hue-angle derivation in actual satellite observations over a large range of natural waters, from the blue oligotrophic ocean waters to the brown peat lakes. A start has been made in the analysis of MERIS and MODIS products from the North Sea in the SNAP environment [23]. It is our hope and expectation that the hue angle parameter will find a place in both ocean-colour science and in citizen science.

Acknowledgments: The research described in this paper was partly funded by the European project Citclops under Grant FP7-ENV-308469. The authors acknowledge the stimulating and fruitful cooperation with all scientists in this wonderful project. We like to thank Marco Peters and Carsten Brockmann from Brockmann Consult for their initiative to convert the hue colour algorithms into a processor in the SNAP software. Hendrik Jan van der Woerd acknowledges A. Dekker for a visitors grant to CSIRO in Canberra, Australia. The opinions expressed in this paper are those of the authors and are not necessarily those of Citclops projects partners or the European Commission.

Author Contributions: Marcel Robert Wernand invented the satellite colour concept and did the handling and quality control of in-situ data. Hendrik Jan van der Woerd develop the algorithms for all sensors. Hendrik Jan van der Woerd and Marcel Robert Wernand wrote the manuscript.

Conflicts of Interest: The authors declare no conflict of interest.

\section{References}

1. Van der Woerd, H.J.; Wernand, M.R. True colour classification of natural waters with medium-spectral resolution satellites: SeaWiFS, MODIS, MERIS and OLCI. Sensors 2015, 15, 25663-25680. [CrossRef] [PubMed]

2. Commission Internationale de l'Éclairage Proceedings, 1931; Cambridge University Press: Cambridge, UK, 1932. 
3. Wyszecki, G.; Stiles, W.S. Color Science: Concepts and Methods, Quantitative Data and Formulae; John Wiley \& Sons: New York, NY, USA, 1982.

4. Citclops (Citizens' Observatory for Coast and Ocean Optical Monitoring). Available online: www.citclops.eu (accessed on 29 July 2017).

5. Busch, J.A.; Badají, R.; Ceccaroni, L.; Friedrichs, A.; Piera, J.; Simon, C.; Thijsse, P.; Wernand, M.; Van der Woerd, H.J.; Zielinski, O. Citizen bio-optical observations from coast- and ocean and their compatibility with ocean colour satellite measurements. Remote Sens. 2016, 8, 879. [CrossRef]

6. Dickinson, J.L.; Shirk, J.; Bonter, D.; Bonney, R.; Crain, R.L.; Martin, J.; Phillips, T.; Purcell, K. The current state of citizen science as a tool for ecological research and public engagement. Front. Ecol. Environ. 2012, 10, 291-297. [CrossRef]

7. Tulloch, A.I.T.; Possingham, H.P.; Joseph, L.N.; Szabo, J.; Martin, T.G. Realising the full potential of citizen science monitoring programs. Biol. Conserv. 2013, 165, 128-138. [CrossRef]

8. Thiel, M.; Penna-Diaz, M.A.; Luna-Jorquera, G.; Salas, S.; Sellanes, J.; Stotz, W. Citizen Scientists and Marine Research: Volunteer participants, their contributions and projection for the future. Oceanogr. Mar. Biol. Annu. Rev. 2014, 52, 257-314.

9. Wernand, M.R.; Van der Woerd, H.J.; Gieskes, W.W.C. Trends in ocean colour and chlorophyll concentration from 1889 to present. PLOS ONE 2013. [CrossRef]

10. Novoa, S.; Wernand, M.R.; Van der Woerd, H.J. The Forel-Ule scale revisited spectrally: Preparation protocols, transmission measurements and chromaticity. J. Eur. Opt. Soc. Rapid Publ. 2013, 8. [CrossRef]

11. Novoa, S.; Wernand, M.R.; Van der Woerd, H.J. The Modern Forel-Ule scale: A 'Do-it-yourself' colour comparator for water monitoring. J. Eur. Opt. Soc. Rapid Publ. 2014, 9. [CrossRef]

12. Mascarenhas, V.J.; Voss, D.; Wollschlaeger, J.; Zielinski, O. Fjord light regime: Bio-optical variability, absorption budget, and hyperspectral light availability in Sognefjord and Trondheimsfjord, Norway. J. Geophys. Res. 2017, 122, 3828-3847. [CrossRef]

13. Novoa, S.; Wernand, M.R.; Van der Woerd, H.J. WACODI: A generic algorithm to derive the intrinsic color of natural waters from digital images. Limnol. Oceanogr. Methods 2015, 13, 697-711. [CrossRef]

14. Busch, J.A.; Price, I.; Jeansou, E.; Zielinski, O.; Van der Woerd, H.J. Citizens and satellites: Assessment of phytoplankton dynamics in a NW Mediterranean aquaculture zone. Int. J. Appl. Earth Obs. Geoinf. 2016, 47, 40-49. [CrossRef]

15. SNAP (Sentinels Application Platform). Available online: http://step.esa.int/main/download/ (accessed on 8 August 2017).

16. Morel, A.; Huot, Y.; Gentili, B.; Werdell, P.J.; Hooker, S.B.; Franz, B.A. Examining the consistency of products derived from various ocean color sensors in open ocean (Case 1) waters in the perspective of a multi-sensor approach. Remote Sens. Environ. 2007, 111, 69-88. [CrossRef]

17. IOCCG. Remote Sensing of Inherent Optical Properties: Fundamentals, Tests of Algorithms, and Applications; Reports of the International Ocean-Colour Coordinating Group, No. 5; Lee, Z.-P., Ed.; IOCCG: Dartmouth, NS, Canada, 2006.

18. Wang, S.; Li, J.; Shen, Q.; Zhang, B.; Zhang, F.; Lu, Z. MODIS-based Radiometric color extraction and classification of inland water with the Forel-Ule scale: A case study of lake Taihu. IEEE J. Sel. Top. Appl. Earth Obs. Remote Sens. 2015, 8, 907-918. [CrossRef]

19. Olmanson, L.G.; Bauer, M.E.; Brezonik, P.L. A 20-year Landsat water clarity census of Minnesota's 10,000 lakes. Remote Sens. Environ. 2008, 112, 4086-4097. [CrossRef]

20. Kutser, T. The possibility of using the Landsat image archive for monitoring long time trends in coloured dissolved organic matter concentration in lake waters. Remote Sens. Environ. 2012, 123, 334-338. [CrossRef]

21. Vanhellemont, Q.; Ruddick, K. Turbid wakes associated with offshore wind turbines observed with Landsat 8. Remote Sens. Environ. 2014, 145, 105-115. [CrossRef]

22. Lymburner, L.; Botha, B.; Hestir, E.; Anstee, J.; Sagar, S.; Dekker, A.; Malthus, T. Landsat 8: Providing continuity and increased precision for measuring multi-decadal time series of total suspended matter. Remote Sens. Environ. 2016, 185, 108-118. [CrossRef]

23. Van der Woerd, H.J.; Wernand, M.; Peters, M.; Bala, M.; Brochmann, C. True color analysis of natural waters with SeaWiFS, MODIS, MERIS and OLCI by SNAP. In Proceedings of the Ocean Optics XXIII, Victoria, BC, Canada, 23-28 October 2016. 
24. Antoine, D.; Morel, A.; Gordon, H.R.; Banzon, V.F.; Evans, R.H. Bridging ocean color observations of the 1980's and 2000's in search of long-term trends. J. Geophys. Res. 2005, 110. [CrossRef]

25. Drusch, M.; Del Bello, U.; Carlier, S.; Colin, O.; Fernandez, V.; Gascon, F.; Bargellini, P. Sentinel-2: ESA's optical high-resolution mission for GMES operational services. Remote Sens. Environ. 2012, 120, 25-36. [CrossRef]

26. Pahlevan, N.; Lee, Z.; Wei, J.; Schaaf, C.B.; Schott, J.R.; Berk, A. On-orbit radiometric characterization of OLI (Landsat-8) for applications in aquatic remote sensing. Remote Sens. Environ. 2014, 154, 272-284. [CrossRef]

27. Ocean Optics Book. Available online: www.oceanopticsbook.info/view/overview-of-optical-oceanography/ reflectances (accessed on 29 July 2017).

28. Mobley, C.D. Hydrolight 3.0 Users' Guide; SRI International: Menlo Park, CA, USA, 1995.

29. IOCCG Data. Available online: http://www.ioccg.org/groups/OCAG_data.html (accessed on 10 August 2015).

30. Mueller, J.L.; Fargion, G.S.; Mcclain, C.R.; Mueller, J.L.; Morel, A.; Frouin, R.; Davis, C.; Arnone, R.; Carder, K.; Steword, R.G.; et al. NASA/TM-2003-Ocean Optics Protocols for Satellite Ocean Color Sensor Validation, Revision 4, Volume III: Radiometric Measurements and Data Analysis Protocols; NASA: Washington DC, USA, 2003.

31. Sentinel-2 SRF. 2017. Available online: https:/ / earth.esa.int/web/sentinel/user-guides/sentinel-2-msi/ document-library/ (accessed on 20 October 2017).

32. USGS Spectral Viewer. Available online: https://landsat.usgs.gov/using-usgs-spectral-viewer (accessed on 30 October 2017).

33. MODIS Relative Spectral Response. Available online: https://mcst.gsfc.nasa.gov/calibration/parameters (accessed on 30 October 2017).

34. Maul, G.A. Introduction to Satellite Oceanography; Martinus Nijhof Publishers: Dordrecht, The Netherlands, 1985.

35. Twardowski, M.S.; Boss, E.; Sullivan, J.M.; Donaghay, P.L. Modeling the spectral shape of absorption by chromophoric dissolved organic matter. Mar. Chem. 2004, 89, 69-88. [CrossRef]

36. Lee, Z.P.; Du, K.P.; Arnone, R. A model for the diffuse attenuation coefficient of downwelling irradiance. J. Geophys. Res. 2005, 110. [CrossRef]

37. Pekel, J.-F.; Cottam, A.; Gorelick, N.; Belward, A.S. High-resolution mapping of global surface water and its long-term changes. Nature 2016, 540, 418-422. [CrossRef] [PubMed]

38. Doxaran, D.; Castaign, P.; Lavender, S.J. Monitoring the maximum turbidity zone and detecting fine-scale turbidity features in the Gironde estuary using high spatial resolution satellite sensor (SPOT HRV, Landsat ETM+) data. Int. J. Remote Sens. 2006, 27, 2303-2321. [CrossRef] 